\title{
Improvement of CEP Accuracy for Vehicles Re-entry Using INS/GPS/GNSS Integrated System
}

\author{
Hadia M. S. EL- Hennawy*, Ibrahim I. Arafa ${ }^{\dagger}$, Alaa M. Fekry*
}

\begin{abstract}
This paper describes the theoretical and practical stages through development to testing of an integrated navigation system, specifically composed of an Inertial Navigation System (INS), and Global Positioning System (GPS). Among various INS/GPS integration strategies, our aim is to construct a mathematical model of INS and GPS systems. A Kalman filter is designed and implemented depending upon these models. Besides these, the performance of the developed system is evaluated with real data recorded by the sensors. A comparison with a reference system and also with a loosely coupled system is done to show the Superiority of the tightly coupled structure. The strap down inertial navigation system (SINS) uses (ENU) frame and the integrated system uses position and velocity as measurements. The system model of the integrated system for Kalman filtering are derived and modeled as 27states. These states estimation system showed clearly the application of fundamental modeling and filtering techniques. The simulation is built on the integrated system INS/GPS/GNSS and the trajectory generator data for flight simulation to be used as a base line algorithm contributing for design, analysis and develop a guidance and control strategy which compensates for the effects of the uncertainties accompanying the navigation systems produced by INS alignment.
\end{abstract}

Keywords: Inertial Navigation Systems and Kalman Filtering, GPS, Initial Alignment

\section{Introduction}

Initial alignment is a key technology for strap down inertial navigation system (SINS), and its precision influences the precision of the navigation system directly [1]. Classical linear error model and standard Kalman filter method cannot solve the problem of SINS initial alignment with large misalignment angles [2]. Therefore, in many application fields with large initial misalignment angles, the nonlinear error model and nonlinear filtering method should be used for the SINS initial alignment with large misalignment angles. One kind of nonlinear error model of SINS with large initial misalignment angles was established in [3]. In [4], the SINS initial alignment was completed by a widely used nonlinear filtering method known as extend Kalman filter (EKF). In existing INS/GPS, evaluation of parameters that characterize the state vector of the air vehicle is performed via extended Kalman filtration. The results of evaluation are used for adjustments of SINS. However, despite the appeal of system integration on the base of Kalman filter, it requires a significant amount of Control Processor Unit (CPU) time to perform the calculations. In addition, according to a number of scientists, there are problems with on-board implementation of the filter. Main one among these problems is the phenomena

Ain Shams University, Cairo, Egypt.

Egyptian Armed Forces, Egypt.

Egyptian Armed Forces, Egypt; ala28444@gmail.com 
of divergence. Also, the main sources of errors in SINS are drifts of gyroscopic sensors and accelerometers, which, due to non-stationary matrix of transition from one coordinate system associated with the air vehicle to another - the navigational one, become non-stationary stochastic processes. This fact creates additional difficulties in identifying SINS errors by the optimal filtering methods [1]. To overcome these problems, a series of modifications of the Kalman filter were developed, in particular, robust and adaptive filtering algorithms, Jazvinskiy algorithm, etc. To reduce the amount of CPU time, the reduced Kalman filter is used, while unobservable parameters of the state vector, in particular, parameters of the angular orientation are corrected from additional sources of information, using, e.g., magnetometers, accelerometers, and other methods of measuring these parameters. Other methods utilize integration of different systems, which works well in practice, particularly in the Dopplerinertial navigation systems. Thus, studies aimed at the improving algorithms of integration of GPS and INS systems are very actual for in-flight mode.

\section{Problem Statement}

The paper suggests the fusion of navigation information on the air vehicle board not on the basis of the reduced Kalman filter, but using the compensation scheme. Similar to implementation based on the Kalman filter, the error estimates from the compensation circuit are used to correct navigation parameters of INS/GPS in flight mode, and external correction is utilized for azimuth and vertical channels of INS/GPS and parameters of angular orientation. Implementation of the Kalman filter requires an extrapolation of errors of the navigation system being corrected, which allows in case of INS malfunctions maintain accuracy characteristics of INS at the right level for some time. Therefore, the problem is stated as the development of algorithms for extrapolation of SINS errors in the compensation circuit, which provide the required accuracy of autonomous functioning of INS when GPS signal is no available.

\section{Problem Solution}

The scheme (Fig. 1) and the algorithm of information processing, which uses the method of compensation, has relatively simple form comparing to the optimal Kalman filtering [1]:

$$
\widehat{X}=X_{S I N S}-F(p)\left(X_{S I N S}-X_{S N S}\right),
$$

Where XSINS, XSNS are navigation parameters (position and velocity components) generated by SINS and GPS; $X$ is the evaluation navigation parameter $X$. For the compensation circuit, a dynamic filter was synthesized, using the procedure which utilizes the regularization technique [2]. It has the following form:

$$
F(p)=\frac{3 T p+1}{(T p+1)(T p+1)(T p+1)}
$$

Structure of the synthesized filter of the compensation circuit is given in Fig. 2.

The system model of the integrated system for Kalman filtering are derived and modeled as 27states. These states estimation system showed clearly the application of fundamental modeling and filtering techniques. The simulation is built on the integrated system INS/GPS/GNSS and the trajectory generator data for flight simulation to be used as a base line algorithm contributing for design, analysis and develop a guidance and control strategy for improving the Air vehicle C.E.P. 


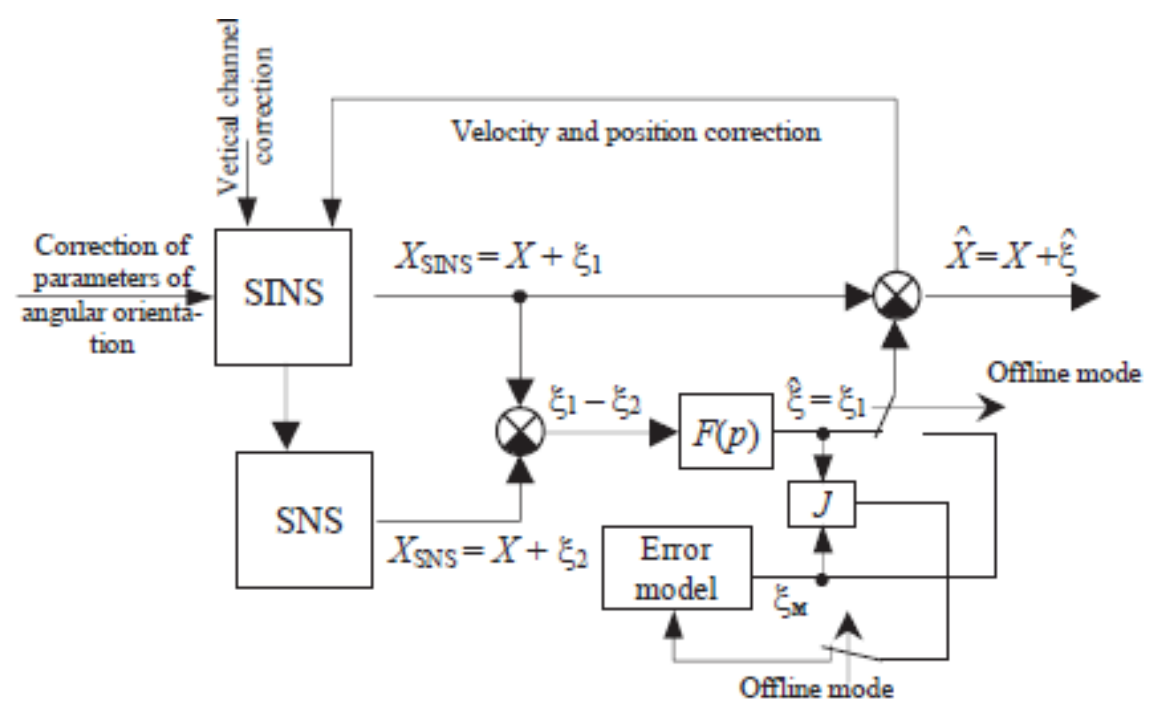

Fig.1 Block diagram of information integrated processing by compensation method

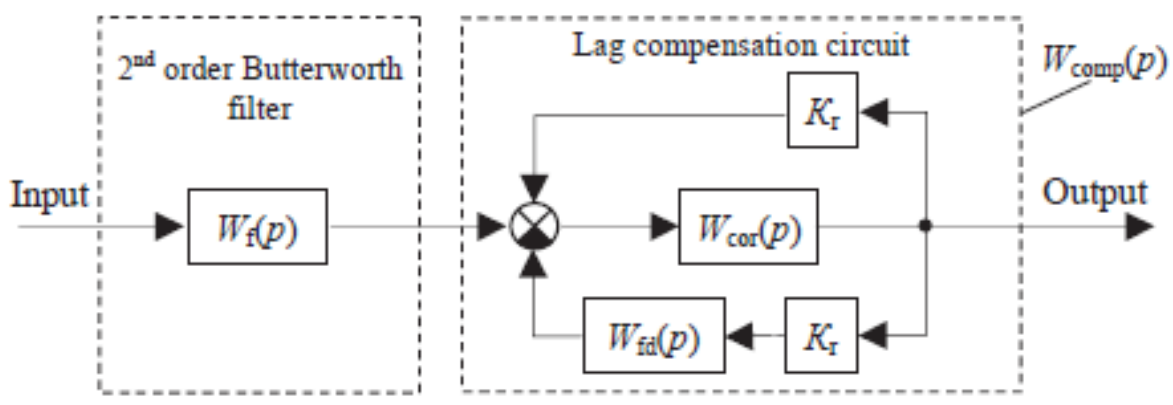

Fig. 2 Compensation circuit filter

Here $W f d(p)=W f(p)$ is the feedback transfer function of the lag compensation circuit; $K r$ is the regularization parameter; $W \operatorname{cor}(p)$ is the transfer function of synthesized correction block; $\operatorname{Wcomp}(p)$ is the transfer function of the lag compensation circuit. Such a filter passes interference $\xi 1$ (the low-frequency error of SINS) with a minimal distortion and damps the interference $\xi 2$ (the high frequency error of SNS), thus making the integrated system error minimal. In the case when frequency characteristics of interferences $\xi 1,, \xi 2$ differ significantly, which takes place in the INS/GPS, the output of the filter $F(p)$ will fully recreate interference $\xi 1$, that is, the lowfrequency error of SINS.

\section{Model of GPS/INS Integrated System}

This section will demonstrate the integration of sensor and system dynamic data and their appropriate models using an optimal filter to create a robust, adaptable, easily reconfigurable state estimation system. This system acquires both global position data and inertial body data, to provide optimal estimates of current position and attitude states. The optimal states are estimated using a Kalman filter. The state estimation system will include three error models for GPS, INS, and instrumentation sensor [81,94,95].

Then, a twenty-seven state Kalman filter integration will be implemented in flight test mode to evaluate system performance. The filter integration approaches will evaluate using data during flight test. The flight test data consisted of measurement of IMU, GPS, and trajectory generator data $[81,95]$. 


\subsection{INS Error Model}

\subsubsection{Position error model}

The geographic implementation of the inertial navigation position rate equations calculates latitude, longitude rate directly as a function of computed missile north and east velocity components in the navigation frame NEU as follows:

$\dot{\varphi}=\frac{\mathrm{V}_{\mathrm{y}}^{\mathrm{n}}}{\mathrm{R}_{\mathrm{M}}+\mathrm{h}} \quad ; \quad \dot{\lambda}=\frac{\mathrm{V}_{\mathrm{x}}^{\mathrm{n}}}{\left(\mathrm{R}_{\mathrm{N}}+\mathrm{h}\right)} \sec \varphi \quad ; \quad \dot{\mathrm{h}}=\mathrm{V}_{\mathrm{z}}^{\mathrm{n}}$

The position error can be modeled as follows:

$\delta \dot{\varphi}=\frac{\delta \mathrm{V}_{\mathrm{y}}^{\mathrm{n}}}{\mathrm{R}_{\mathrm{M}}+\mathrm{h}} ; \delta \dot{\lambda}=\frac{\delta \mathrm{V}_{\mathrm{x}}^{\mathrm{n}}}{\left(\mathrm{R}_{\mathrm{N}}+\mathrm{h}\right) \cos \varphi}+\frac{\mathrm{V}_{\mathrm{x}}^{\mathrm{n}} \tan \varphi \sec \varphi}{\mathrm{R}_{\mathrm{N}}+\mathrm{h}} \delta \varphi ; \delta \dot{\mathrm{h}}=\delta \mathrm{V}_{\mathrm{z}}^{\mathrm{n}}$

4.1.2 Attitude error model

The attitude error model using Euler angle can be written as:

$\dot{\varphi}^{\mathrm{n}}=\varphi^{\mathrm{n}} \times \omega_{\mathrm{in}}^{\mathrm{n}}+\delta \omega_{\mathrm{in}}^{\mathrm{n}}-\mathrm{C}_{\mathrm{b}}^{\mathrm{n}} \delta \omega_{\mathrm{ib}}^{\mathrm{b}}$

This equation can be rewritten as:

$$
\begin{aligned}
\dot{\varphi}^{n} & =-\omega_{i n}^{n} \times \varphi^{n}+\delta \omega_{i n}^{n}-C_{b}^{n} \delta \omega_{i b}^{b} \\
& =-\left(\omega_{i e}^{n}+\omega_{e n}^{n}\right) \times \varphi^{n}+\delta \omega_{i e}^{n}+\delta \omega_{e n}^{n}+\Delta \omega^{n}
\end{aligned}
$$

where $\delta \omega_{i b}^{n}$ is the error in measuring angular velocity vector due to gyro errors from body frame to inertial frame resolved in the navigation frame and can be define as:

$\Delta \omega^{n}=C_{b}^{n} \delta \omega_{i b}^{n}$

From the standard navigation mechanization in ENU frame we get:

$\omega_{i e}^{n}=\left[\begin{array}{lll}0 & \Omega \cos \varphi & \Omega \sin \varphi\end{array}\right]^{\mathrm{T}}$

$\delta \omega_{i e}^{n}=\left[\begin{array}{lll}0 & -\Omega \sin \varphi \delta \varphi & \Omega \cos \varphi \delta \varphi\end{array}\right]^{\mathrm{T}}$

$\boldsymbol{\omega}_{e n}^{n}=\left[\begin{array}{lll}\frac{-\mathrm{V}_{\mathrm{y}}^{\mathrm{n}}}{\mathrm{R}_{\mathrm{M}}+\mathrm{h}} & \frac{\mathrm{V}_{\mathrm{x}}^{\mathrm{n}}}{\mathrm{R}_{\mathrm{N}}+\mathrm{h}} & \frac{\mathrm{V}_{\mathrm{x}}^{\mathrm{n}} \tan \varphi}{\mathrm{R}_{\mathrm{N}}+\mathrm{h}}\end{array}\right]^{\mathrm{T}}$

$\delta \omega_{e n}^{n}=\left[\begin{array}{c}\frac{-\delta \mathrm{V}_{\mathrm{y}}^{\mathrm{n}}}{\mathrm{R}_{\mathrm{M}}+\mathrm{h}}+\frac{\mathrm{V}_{\mathrm{y}}^{\mathrm{n}}}{\left(\mathrm{R}_{\mathrm{M}}+\mathrm{h}\right)^{2}}\left(\delta \mathrm{R}_{\mathrm{M}}+\delta \mathrm{h}\right) \\ \frac{\delta \mathrm{V}_{\mathrm{x}}^{\mathrm{n}}}{\mathrm{R}_{\mathrm{N}}+\mathrm{h}}-\frac{\mathrm{V}_{\mathrm{x}}^{\mathrm{n}}}{\left(\mathrm{R}_{\mathrm{N}}+\mathrm{h}\right)^{2}}\left(\delta \mathrm{R}_{\mathrm{N}}+\delta \mathrm{h}\right) \\ \frac{\delta \mathrm{V}_{\mathrm{x}}^{\mathrm{n}} \tan \varphi}{\mathrm{R}_{\mathrm{N}}+\mathrm{h}}-\frac{\mathrm{V}_{\mathrm{x}}^{\mathrm{n}} \tan \varphi}{\left(\mathrm{R}_{\mathrm{N}}+\mathrm{h}\right)^{2}}\left(\delta \mathrm{R}_{\mathrm{N}}+\delta \mathrm{h}\right)+\frac{\mathrm{V}_{\mathrm{x}}^{\mathrm{n}} \sec ^{2} \varphi}{\mathrm{R}_{\mathrm{N}}+\mathrm{h}} \delta \varphi\end{array}\right]$ 
From (5) (.8) and (4), the attitude error components can be written as [22]:

$$
\begin{gathered}
\dot{\phi}_{\mathrm{x}}^{\mathrm{n}}=-\frac{\delta \mathrm{V}_{\mathrm{y}}^{\mathrm{n}}}{\mathrm{R}_{\mathrm{M}}+\mathrm{h}}+\left(\Omega \sin \varphi+\frac{\mathrm{V}_{\mathrm{x}}^{\mathrm{n}} \tan \varphi}{\mathrm{R}_{\mathrm{N}}+\mathrm{h}}\right) \phi_{\mathrm{y}}^{\mathrm{n}}-\left(\Omega \cos \varphi+\frac{\mathrm{V}_{\mathrm{x}}^{\mathrm{n}}}{\mathrm{R}_{\mathrm{N}}+\mathrm{h}}\right) \phi_{\mathrm{z}}^{\mathrm{n}}+\Delta \omega_{\mathrm{x}}^{\mathrm{n}} \\
\dot{\phi}_{\mathrm{y}}^{\mathrm{n}}=-\Omega \sin \varphi \delta \varphi+\frac{\delta \mathrm{V}_{\mathrm{x}}^{\mathrm{n}}}{\mathrm{R}_{\mathrm{M}}+\mathrm{h}}-\left(\Omega \sin \varphi+\frac{\mathrm{V}_{\mathrm{x}}^{\mathrm{n}} \tan \varphi}{\mathrm{R}_{\mathrm{N}}+\mathrm{h}}\right) \phi_{\mathrm{x}}^{\mathrm{n}}-\frac{\mathrm{V}_{\mathrm{y}}^{\mathrm{n}}}{\mathrm{R}_{\mathrm{M}}+\mathrm{h}} \phi_{\mathrm{z}}^{\mathrm{n}}+\Delta \omega_{\mathrm{y}}^{\mathrm{n}} \\
\dot{\phi}_{\mathrm{z}}^{\mathrm{n}}=\left(\Omega \cos \varphi+\frac{\mathrm{V}_{\mathrm{x}}^{\mathrm{n}} \sec ^{2} \varphi}{\mathrm{R}_{\mathrm{N}}+\mathrm{h}}\right) \delta \varphi+\frac{\tan \varphi}{\mathrm{R}_{\mathrm{N}}+\mathrm{h}} \delta \mathrm{V}_{\mathrm{x}}^{\mathrm{n}}+\left(\Omega \cos \varphi+\frac{\mathrm{V}_{\mathrm{x}}^{\mathrm{n}}}{\mathrm{R}_{\mathrm{N}}+\mathrm{h}}\right) \phi_{\mathrm{x}}^{\mathrm{n}}+ \\
\frac{\mathrm{V}_{\mathrm{y}}^{\mathrm{n}}}{\mathrm{R}_{\mathrm{M}}+\mathrm{h}} \phi_{\mathrm{y}}^{\mathrm{n}}+\Delta \omega_{\mathrm{z}}^{\mathrm{n}}
\end{gathered}
$$

where $\Delta \omega_{x}^{n}, \Delta \omega_{y}^{n}, \Delta \omega_{z}^{n}$ are the angular velocity errors in navigation frame due to gyro measurement errors.

4.1.3 Velocity error model

The SINS true velocity error in navigation frame is given by:

$\dot{\mathbf{V}}^{\mathrm{n}}=\mathbf{C}_{\mathrm{b}}^{\mathrm{n}} \mathbf{f}^{\mathrm{b}}-\left(2 \boldsymbol{\omega}_{\mathrm{ie}}^{\mathrm{n}}+\boldsymbol{\omega}_{\mathrm{en}}^{\mathrm{n}}\right) \times \mathbf{V}^{\mathrm{n}}+\mathbf{g}^{\mathrm{n}}(12)$

This equation can be rewrite as:

$$
\dot{\mathbf{V}}^{\mathbf{n}}=\mathbf{f}^{\mathbf{n}}-\left(2 \boldsymbol{\omega}_{\mathrm{ie}}^{\mathrm{n}}+\boldsymbol{\omega}_{\mathrm{en}}^{\mathrm{n}}\right) \times \mathbf{V}^{\mathrm{n}}+\mathbf{g}^{\mathrm{n}}
$$

The velocity error equation can be written as:

$$
\delta \dot{V}^{n}=\delta \mathbf{f}^{n}-\left(2 \delta \omega_{i e}^{n}+\delta \omega_{e n}^{n}\right) \times V^{n}-\left(2 \omega_{i e}^{n}+\omega_{e n}^{n}\right) \times \delta V^{n}+\delta g^{n}
$$

where $\delta \boldsymbol{g}^{n}=0$ and,

$$
\begin{aligned}
\delta \mathbf{f}^{\mathrm{n}} & =\hat{\mathbf{f}}^{\mathrm{n}}-\mathbf{f}^{\mathrm{n}}=\hat{\mathbf{C}}_{\mathrm{b}}^{\mathrm{n}} \hat{\mathbf{f}}^{\mathrm{b}}-\mathbf{f}^{\mathrm{n}}=(\mathbf{I}-\Phi) \mathbf{C}_{\mathrm{b}}^{\mathrm{n}}\left(\mathbf{f}^{\mathrm{b}}+\Delta \mathbf{f}^{\mathrm{b}}\right)-\mathbf{f}^{\mathrm{n}} \\
& \approx(\mathbf{I}-\Phi) \mathbf{f}^{\mathrm{n}}+\Delta \mathbf{f}^{\mathrm{n}}-\mathbf{f}^{\mathrm{n}}=\mathbf{f}^{\mathrm{n}} \times \boldsymbol{\varphi}^{\mathrm{n}}+\Delta \mathbf{f}^{\mathrm{n}}
\end{aligned}
$$

Then (14) can be rewrite as:

$$
\delta \dot{V}^{n}=\mathbf{f}^{n} \times \varphi^{n}-\left(2 \delta \omega_{i e}^{n}+\delta \omega_{e n}^{n}\right) \times V^{n}-\left(2 \omega_{i e}^{n}+\omega_{e n}^{n}\right) \times \delta V^{n}+\Delta \mathbf{f}^{n}
$$

and the velocity error components are given by:

$$
\begin{aligned}
\delta \dot{\mathrm{V}}_{\mathrm{x}}^{\mathrm{n}}=\mathrm{f}_{\mathrm{y}}^{\mathrm{n}} \phi_{\mathrm{z}}^{\mathrm{n}}-\mathrm{f}_{\mathrm{z}}^{\mathrm{n}} \phi_{\mathrm{y}}^{\mathrm{n}}+\left(2 \Omega \mathrm{V}_{\mathrm{y}}^{\mathrm{n}} \cos \varphi+2 \Omega \mathrm{V}_{\mathrm{z}}^{\mathrm{n}} \sin \varphi+\frac{\mathrm{V}_{\mathrm{x}}^{\mathrm{n}} \mathrm{V}_{\mathrm{y}}^{\mathrm{n}} \sec ^{2} \varphi}{\mathrm{R}_{\mathrm{N}}+\mathrm{h}}\right) \delta \varphi+ \\
\frac{\mathrm{V}_{\mathrm{y}}^{\mathrm{n}} \tan \varphi-\mathrm{V}_{\mathrm{z}}^{\mathrm{n}}}{\mathrm{R}_{\mathrm{N}}+\mathrm{h}} \delta \mathrm{V}_{\mathrm{x}}^{\mathrm{n}}+\left(2 \Omega \sin \varphi+\frac{\mathrm{V}_{\mathrm{x}}^{\mathrm{n}} \tan \varphi}{\mathrm{R}_{\mathrm{N}}+\mathrm{h}}\right) \delta \mathrm{V}_{\mathrm{y}}^{\mathrm{n}}- \\
\left(2 \Omega \cos \varphi+\frac{\mathrm{V}_{\mathrm{x}}^{\mathrm{n}}}{\mathrm{R}_{\mathrm{N}}+\mathrm{h}}\right) \delta \mathrm{V}_{\mathrm{z}}^{\mathrm{n}}+\Delta \mathrm{f}_{\mathrm{x}}^{\mathrm{n}}
\end{aligned}
$$




$$
\begin{gathered}
\delta \dot{\mathrm{V}}_{\mathrm{y}}^{\mathrm{n}}=\mathrm{f}_{\mathrm{z}}^{\mathrm{n}} \phi_{\mathrm{x}}^{\mathrm{n}}-\mathrm{f}_{\mathrm{x}}^{\mathrm{n}} \phi_{\mathrm{z}}^{\mathrm{n}}-\left(2 \Omega \mathrm{V}_{\mathrm{x}}^{\mathrm{n}} \cos \varphi+\frac{\left(\mathrm{V}_{\mathrm{x}}^{\mathrm{n}}\right)^{2} \sec ^{2} \varphi}{\mathrm{R}_{\mathrm{N}}+\mathrm{h}}\right) \delta \varphi- \\
\left(2 \Omega \sin \varphi+\frac{2 \mathrm{~V}_{\mathrm{x}}^{\mathrm{n}} \tan \varphi}{\mathrm{R}_{\mathrm{N}}+\mathrm{h}}\right) \delta \mathrm{V}_{\mathrm{x}}^{\mathrm{n}}-\frac{\mathrm{V}_{\mathrm{z}}^{\mathrm{n}}}{\mathrm{R}_{\mathrm{M}}+\mathrm{h}} \delta \mathrm{V}_{\mathrm{y}}^{\mathrm{n}}-\frac{\mathrm{V}_{\mathrm{z}}^{\mathrm{n}}}{\mathrm{R}_{\mathrm{M}}+\mathrm{h}} \delta \mathrm{V}_{\mathrm{z}}^{\mathrm{n}}+\Delta \mathrm{f}_{\mathrm{y}}^{\mathrm{n}} \\
\delta \dot{\mathrm{V}}_{\mathrm{z}}^{\mathrm{n}}=\mathrm{f}_{\mathrm{x}}^{\mathrm{n}} \phi_{\mathrm{y}}^{\mathrm{n}}-\mathrm{f}_{\mathrm{y}}^{\mathrm{n}} \phi_{\mathrm{x}}^{\mathrm{n}}-2 \Omega \mathrm{V}_{\mathrm{x}}^{\mathrm{n}} \sin \varphi \delta \varphi+\left(2 \Omega \cos \varphi+\frac{2 \mathrm{~V}_{\mathrm{x}}^{\mathrm{n}}}{\mathrm{R}_{\mathrm{N}}+\mathrm{h}}\right) \delta \mathrm{V}_{\mathrm{x}}^{\mathrm{n}}+ \\
\frac{2 \mathrm{~V}_{\mathrm{y}}^{\mathrm{n}}}{\mathrm{R}_{\mathrm{M}}+\mathrm{h}} \delta \mathrm{V}_{\mathrm{y}}^{\mathrm{n}}+\Delta \mathrm{f}_{\mathrm{z}}^{\mathrm{n}}
\end{gathered}
$$

where $\Delta \mathrm{f}_{\mathrm{x}}^{\mathrm{n}}, \Delta \mathrm{f}_{\mathrm{y}}^{\mathrm{n}}, \Delta \mathrm{f}_{\mathrm{z}}^{\mathrm{n}}$ are the errors in the specific acceleration due to accelerometers error in navigation frame.

\subsubsection{GPS/GLONASS error model}

Now we suppose that the output from GPS/GLONASS in the frame of WGS-84. In many literatures the GPS/GLONASS have a lot of error sources which effects on the accuracy of position and velocity. Even more, if most of these errors are corrected, still some random errors are existing. We can regard the random error as clock errors that are the first differential equation which describe the state errors can be written as:

$\dot{\mathbf{x}}_{\mathrm{GPS}}=\mathbf{F}_{\mathrm{GPS}} \mathbf{x}_{\mathrm{GPS}}+\mathbf{w}_{\mathrm{GPS}}$

$\mathbf{x}_{\mathrm{GPS}}=\left[\begin{array}{llllll}\delta \varphi_{\mathrm{GPS}} & \delta \lambda_{\mathrm{GPS}} & \delta \mathrm{h}_{\mathrm{GPS}} & \delta \mathrm{V}_{\mathrm{x}_{\mathrm{GPS}}} & \delta \mathrm{V}_{\mathrm{y}_{\mathrm{GPS}}} & \delta \mathrm{V}_{\mathrm{z}_{\mathrm{GPS}}}\end{array}\right]^{\mathrm{T}}$

where $\delta \varphi_{\mathrm{GPS}}, \delta \lambda_{\mathrm{GPS}}, \delta \mathrm{h}_{\mathrm{GPS}}$ are the position errors of GPS and $\delta \mathrm{V}_{\mathrm{x}_{\mathrm{GPS}}}, \delta \mathrm{V}_{\mathrm{y}_{\mathrm{GPS}}}, \delta \mathrm{V}_{\mathrm{z}_{\mathrm{GPS}}}$ are the velocity errors of GPS. We usually regard the position and velocity errors of GPS as first order Markov process noise, $\tau_{\varphi_{\mathrm{GPS}}}, \tau_{\lambda_{\mathrm{GPS}}}, \tau_{\mathrm{h}_{\mathrm{GPS}}}, \tau_{\mathrm{Vx}_{\mathrm{GPS}}}, \tau_{\mathrm{V}_{\mathrm{G}} \mathrm{GP}}, \tau_{\mathrm{Vz}_{\mathrm{GPS}}}$ are Markov process time constants. Then the GPS error system matrix can be define as:

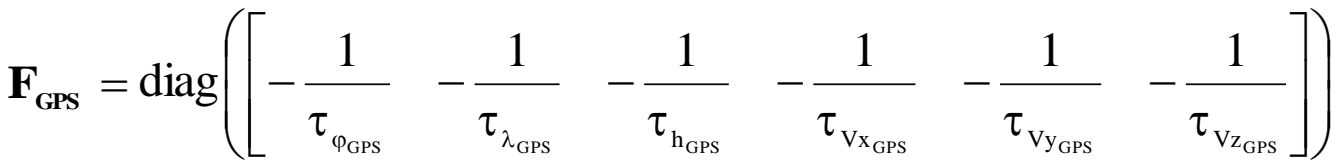

and the GPS noise vector can be define as:

$\mathbf{w}_{\mathrm{GPS}}=\left[\begin{array}{llllll}\omega_{\varphi_{\mathrm{GPS}}} & \omega_{\lambda_{\mathrm{GPS}}} & \omega_{\mathrm{h}_{\mathrm{GPS}}} & \omega_{\mathrm{vx}_{\mathrm{GPS}}} & \omega_{\mathrm{Vy}_{\mathrm{GPS}}} & \omega_{\mathrm{vz}_{\mathrm{GPS}}}\end{array}\right]^{\mathrm{T}}$

where $\omega_{\varphi_{\mathrm{GPS}}}, \omega_{\lambda_{\mathrm{GPS}}}, \omega_{\mathrm{h}_{\mathrm{GPS}}}$ and $\omega_{\mathrm{Vx}_{\mathrm{GPS}}}, \omega_{\mathrm{Vy}_{\mathrm{GPS}}}, \omega_{\mathrm{Vz}_{\mathrm{GPS}}}$ are the white noise in the GPS position and velocity respectively.

\subsection{State and Measurement Equations of Integrated System}

From the above 3-models (INS error model, and GPS/GLONASS error model) we can construct the integration navigation system error model as follows:

$\dot{\boldsymbol{x}}(\mathrm{t})=\boldsymbol{F}(\boldsymbol{t}) \boldsymbol{x}(\mathrm{t})+\boldsymbol{G}(\mathrm{t}) \boldsymbol{w}(\mathrm{t})$ 
where $\boldsymbol{x}(\mathrm{t}) \in \mathfrak{R}^{27}$ is the system state vector, $\boldsymbol{F}(\mathrm{t}) \in \mathfrak{R}^{27 \times 27}$ is the dynamic system matrix, $\boldsymbol{w}(\boldsymbol{t}) \in \mathfrak{R}^{12}$ is the system process noise vector, and $\boldsymbol{G}(\mathrm{t}) \in \mathfrak{R}^{27 \times 12}$ is the system noise matrix. Then the state vector of the INS/GPS/GLONASS integrated system can be written as:

$$
\begin{aligned}
& {\left[\begin{array}{llllllllll}
\phi_{\mathrm{x}}^{\mathrm{p}} & \phi_{\mathrm{y}}^{\mathrm{p}} & \phi_{\mathrm{z}}^{\mathrm{p}} & \delta \varphi & \delta \lambda & \delta \mathrm{h} & \delta \mathbf{V}_{\mathrm{x}}^{\mathrm{p}} & \delta \mathbf{V}_{\mathrm{y}}^{\mathrm{p}} & \delta \mathbf{V}_{\mathrm{z}}^{\mathrm{p}}
\end{array}\right.}
\end{aligned}
$$

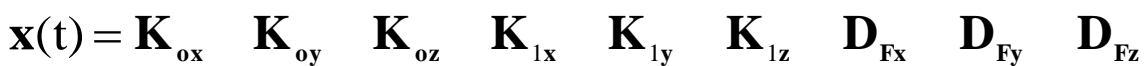

$$
\begin{aligned}
& \left.\begin{array}{lllllllll}
\mathbf{S}_{\mathrm{Gx}} & \mathbf{S}_{\mathrm{Gy}} & \mathbf{S}_{\mathrm{Gz}} & \boldsymbol{\delta}_{\varphi_{\mathrm{GPS}}} & \boldsymbol{\delta}_{\lambda_{\mathrm{GPS}}} & \boldsymbol{\delta}_{\mathrm{h}_{\mathrm{GPS}}} & \boldsymbol{\delta}_{\mathrm{Vx}_{\mathrm{GPS}}} & \boldsymbol{\delta}_{\mathrm{Vy}_{\mathrm{GPS}}} & \boldsymbol{\delta}_{\mathrm{Vz}_{\mathrm{GPS}}}
\end{array}\right]^{\mathrm{T}} \\
& \mathbf{F}(\mathrm{t})=\left[\begin{array}{lll}
\mathbf{F}_{\text {INS }} & \mathbf{F}_{\text {sen. }} & 0_{9 \times 6} \\
0_{12 \times 9} & 0_{12 \times 12} & 0_{12 \times 6} \\
0_{6 \times 9} & 0_{6 \times 12} & \mathbf{F}_{\text {GPS }}
\end{array}\right]
\end{aligned}
$$

where $\boldsymbol{F}_{I N S}$ is the system dynamic matrix of navigation error model, can be constructed as:

$$
\mathbf{F}_{\text {INS }}=\left[\begin{array}{lll}
\mathbf{F}_{11} & \mathbf{F}_{12} & \mathbf{F}_{13} \\
\mathbf{F}_{21} & \mathbf{F}_{22} & \mathbf{F}_{23} \\
0_{3 \times 3} & \mathbf{F}_{32} & \mathbf{F}_{33}
\end{array}\right]
$$

and the system noise vector $\boldsymbol{w}(\mathrm{t})$ can be define as:

$$
\begin{aligned}
& w(t)=
\end{aligned}
$$

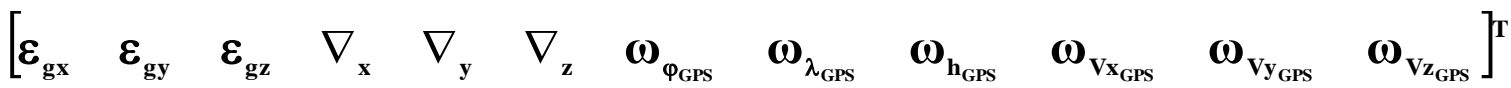

where $\boldsymbol{\varepsilon}_{\mathrm{g}}, \nabla$ are the gyro and accelerometer white noise vectors. The input system noise matrix can also be constructing as follows:

$$
\mathbf{G}(\mathrm{t})=\left[\begin{array}{ccc}
\mathbf{C}_{\mathbf{b}}^{\mathbf{p}} & 0_{3 \times 3} & 0_{3 \times 6} \\
0_{3 \times 3} & \mathbf{C}_{\mathbf{b}}^{\mathbf{p}} & 0_{3 \times 6} \\
0_{3 \times 3} & 0_{3 \times 3} & 0_{3 \times 6} \\
0_{12 \times 3} & 0_{12 \times 3} & 0_{12 \times 6} \\
0_{6 \times 3} & 0_{6 \times 3} & \mathbf{I}_{6 \times 6}
\end{array}\right]
$$

The measurement equation of the integrated INS/GPS/GLONASS navigation systems based on the position and velocity measurements. Then we can use the output from INS/GPS/GLONASS as the measurements. So, the measurement equation can be defined as follows:

$\mathrm{z}=\mathrm{Hx}+\mathrm{v}$

where $\boldsymbol{H}$ is the measurement matrix and can be defined as:

$$
\mathrm{H}=\left[\begin{array}{cccccc}
0_{3 \times 3} & 0_{3 \times 3} & \mathrm{I}_{3 \times 3} & 0_{3 \times 3} & -\mathrm{I}_{3 \times 3} & 0_{3 \times 3} \\
0_{3 \times 3} & \mathrm{I}_{3 \times 3} & 0_{3 \times 3} & 0_{3 \times 3} & 0_{3 \times 3} & -\mathrm{I}_{3 \times 3}
\end{array}\right]
$$

and $\boldsymbol{x}$ is the observed measurement state and can be defied as: 


$$
\mathrm{X}=\left[\begin{array}{c}
\varphi_{\mathrm{INS}}-\varphi_{\mathrm{GPS}} \\
\lambda_{\mathrm{INS}}-\lambda_{\mathrm{GPS}} \\
\mathrm{h}_{\mathrm{INS}}-\mathrm{h}_{\mathrm{GPS}} \\
\mathrm{V}_{\mathrm{x}_{\mathrm{INS}}}-\mathrm{V}_{\mathrm{x}_{\mathrm{GPS}}} \\
\mathrm{V}_{\mathrm{y}_{\mathrm{INS}}}-\mathrm{V}_{\mathrm{y}_{\mathrm{GPS}}} \\
\mathrm{V}_{\mathrm{z}_{\mathrm{INS}}}-\mathrm{V}_{\mathrm{z}_{\mathrm{GPS}}}
\end{array}\right]=\left[\begin{array}{c}
\delta \varphi-\delta_{\varphi_{\mathrm{GPS}}} \\
\delta \lambda-\delta_{\lambda_{\mathrm{GPS}}} \\
\delta \mathrm{h}-\delta_{\mathrm{h}_{\mathrm{GPS}}} \\
\delta \mathrm{V}_{\mathrm{x}}-\delta_{\mathrm{v}_{\mathrm{GPS}}} \\
\delta \mathrm{V}_{\mathrm{y}}-\delta_{\mathrm{V}_{\mathrm{GPS}}} \\
\delta \mathrm{V}_{\mathrm{z}}-\delta_{\mathrm{V}_{\mathrm{GPS}}}
\end{array}\right]
$$

and $\boldsymbol{v} \in \mathfrak{R}^{6}$ is the measurement noise vector.

\subsection{Kalman Filter Functionality and Mechanization}

The flight System Error Estimate (SEE) Utilizes the Kalman filter to generate corrections to the navigation system using the difference between the GPS position, velocity and navigation position, velocity as the measurement [82]. To use Kalman filter for the integration INS/GPS/GLONASS, we must discretize the continuous system matrix equation. The discretization of the state equation and measurement equation are as follows $[22,82,96]$ :

$$
\begin{aligned}
& \mathbf{x}_{k}=\Phi_{k, k-1} x_{k-1}+\Gamma_{k-1} \mathbf{W}_{k-1} \\
& \mathbf{z}_{k}=\mathbf{H}_{k}, \mathbf{x}_{k}+\mathbf{v}_{k}
\end{aligned}
$$

where $\boldsymbol{\Phi}_{k, k-1}$ is the system discrete matrix, $\boldsymbol{\Gamma}_{k-1}$ is the discrete system noise process.

$$
\begin{aligned}
& \boldsymbol{\Phi}_{k, k-1}=\sum_{n=0}^{\infty} \frac{\left[\mathbf{F}\left(t_{k}\right) T\right]^{n}}{n !} \\
& \Gamma_{k-1}=\left\{\sum_{n=1}^{\infty}\left[\frac{1}{n !}\left(\mathbf{F}\left(t_{k}\right) T\right)^{n-1}\right]\right\} \mathbf{G}\left(t_{k}\right) T
\end{aligned}
$$

where $\mathrm{T}$ is the interval time, in the real time we use some definite time.

- Time update

$$
\begin{aligned}
& \hat{\mathbf{x}}_{\mathrm{k} / \mathrm{k}-1}=\boldsymbol{\Phi}_{\mathrm{k}, \mathrm{k}-1} \hat{\mathbf{x}}_{\mathrm{k}-1} \\
& \mathbf{P}_{\mathrm{k} / \mathrm{k}-1}=\boldsymbol{\Phi}_{\mathrm{k}, \mathrm{k}-1} \mathbf{P}_{\mathrm{k}-1} \boldsymbol{\Phi}_{\mathrm{k}, \mathrm{k}-1}^{\mathrm{T}}+\boldsymbol{\Gamma}_{\mathrm{k}-1} \mathbf{Q}_{\mathrm{k}-1} \Gamma_{\mathrm{k}-1}^{\mathrm{T}}
\end{aligned}
$$

- Measurement update

$$
\begin{aligned}
& \mathbf{K}_{\mathrm{k}}=\mathbf{P}_{\mathrm{k} / \mathrm{k}-1} \mathbf{H}_{\mathrm{k}}^{\mathrm{T}}\left(\mathbf{H}_{\mathrm{k}} \mathbf{P}_{\mathrm{k} / \mathrm{k}-1} \mathbf{H}_{\mathrm{k}}^{\mathrm{T}}+\mathbf{R}_{\mathrm{k}}\right)^{-1} \\
& \hat{\mathbf{x}}_{\mathrm{k}}=\hat{\mathbf{x}}_{\mathrm{k} / \mathrm{k}-1}+\mathbf{K}_{\mathrm{k}}\left(\mathbf{z}_{\mathrm{k}}-\mathbf{H}_{\mathrm{k}} \hat{\mathbf{x}}_{\mathrm{k} / \mathrm{k}-1}\right) \\
& \mathbf{P}_{\mathrm{k}}=\left(\mathbf{I}-\mathbf{K}_{\mathrm{k}} \mathbf{H}_{\mathrm{k}}\right) \mathbf{P}_{\mathrm{k} / \mathrm{k}-1}
\end{aligned}
$$




\subsection{Simulation Parameters}

Sampling time of trajectory data is chosen as $10 \mathrm{~ms}$, INS computation interval time $20 \mathrm{~ms}$, GPS/GLONASS transfer data frequency1HZ, and Kalman filter interval time $1 \mathrm{sec}$.

\subsubsection{INS parameters}

The initial attitude error angle is chosen as 500", initial velocity error $1.0 \mathrm{~m} / \mathrm{s}$, initial error of latitude and longitude angle $0.15 \mathrm{rad}$, initial altitude $1000 \mathrm{~m}$, initial accelerometer bias error $10^{-8} \mathrm{~m} / \mathrm{s}^{2}$, initial accelerometer scale factor error $10^{-8}$, initial gyro fixed drift error $10^{-8} \mathrm{n} / \mathrm{s}^{2}$, initial gyro scale factor error $10^{-8}$, random drift of each gyro $0.1^{\circ} / \mathrm{h}$, and random bias of each accelerometer $10 \mu \mathrm{g}$.

\subsubsection{GPS/GLONASS parameters}

Initial velocity error is chosen as $5 \mathrm{~m} / \mathrm{s}$, initial error of latitude and longitude angle $0.0015 \mathrm{rad}$, initial altitude $10 \mathrm{~m}$, Markov time constant $\tau=60 \mathrm{sec}$, random altitude $5.0 \mathrm{~m}$, and random in measuring velocity $1.0 \mathrm{~m} / \mathrm{s}$.

Fig.3. shows the INS estimated errors, and the GPS estimated errors.
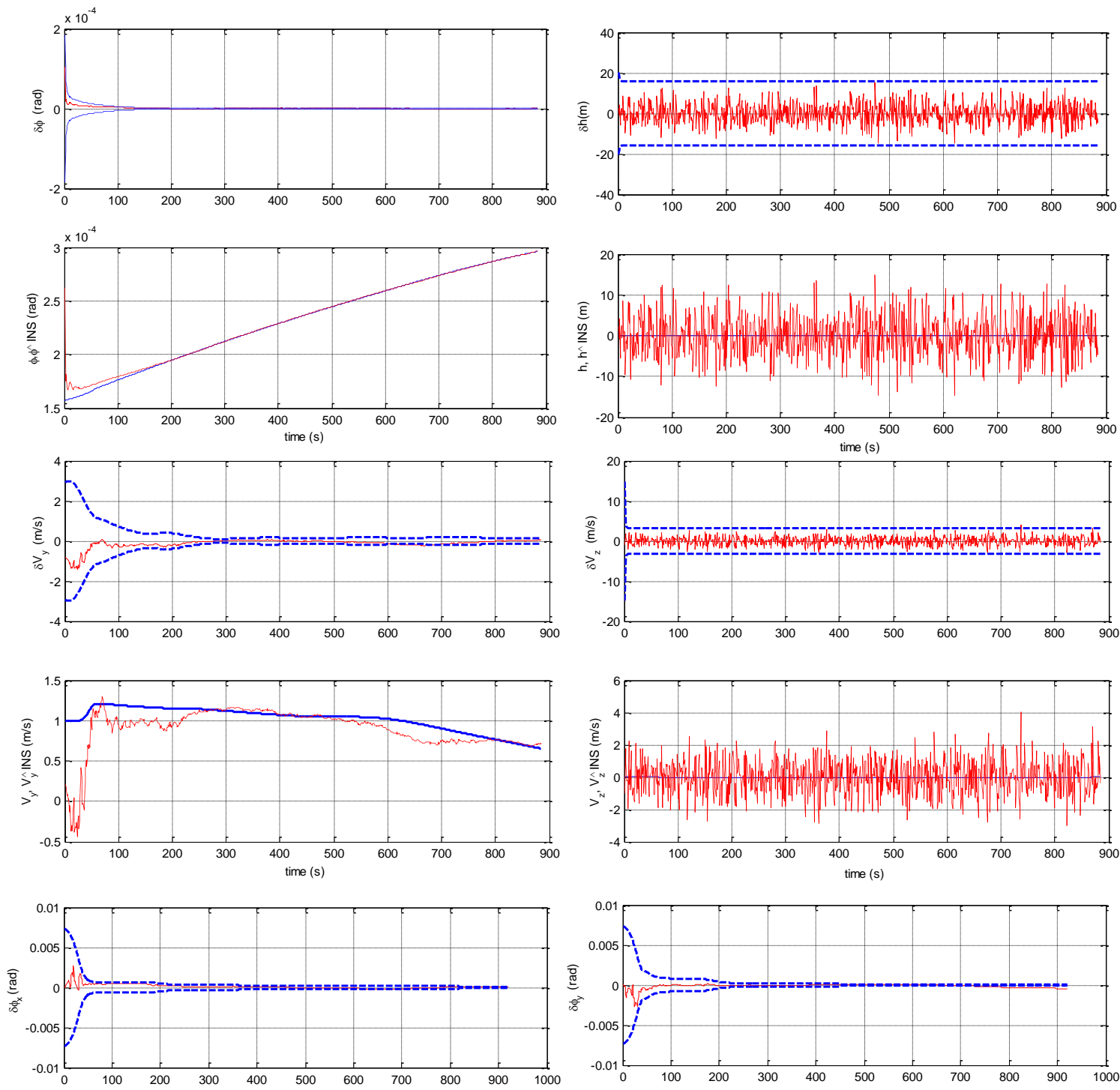

Fig.3. The INS estimated errors (lift) and the GPS estimated errors (right),

(continued next page). 

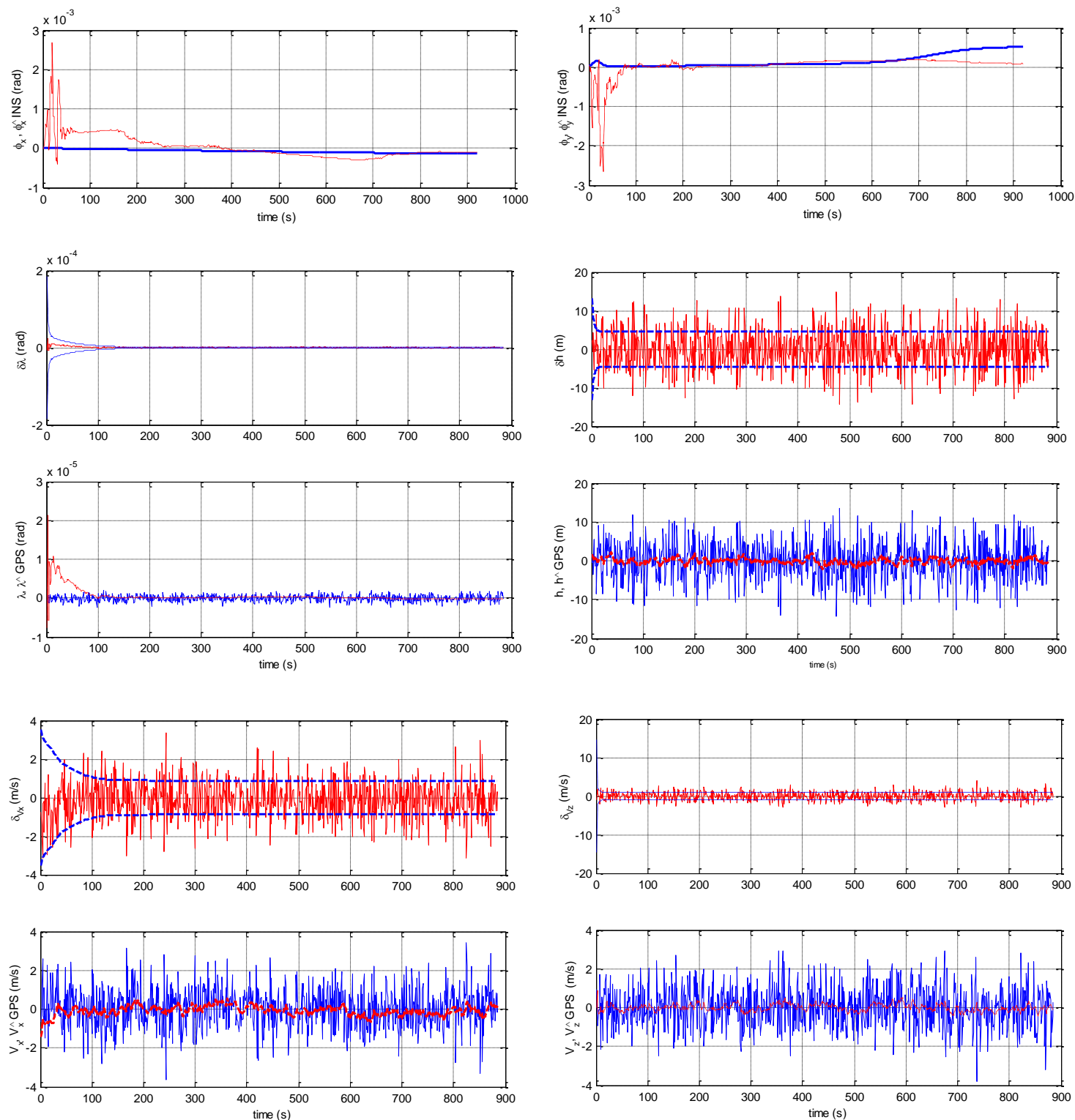

Fig.3 (continued). The INS estimated errors (lift) and the GPS estimated errors (right)

\section{Conclusion}

This paper demonstrated the importance of the INS/GPS/GLONASS integration into ballistic navigation solution. The SINS uses (ENU) frame and the integrated system uses position and velocity as measurements. The system model of the integrated system for Kalman filtering are derived and modeled as 27-states. This state estimation system shown clearly the application of fundamental modeling and filtering techniques. The simulation is built on the integrated system INS/GPS/GLONASS as shown in fig (5.5) and the trajectory generator data. From the results we found that some parameters of estimated gyro errors such as vertical and east gyro drifts, and also estimated east accelerometer bias are not observables. 
The simulation shows that in the integrated system, the navigation errors in both the INS and GPS/GLONASS can be estimated with high accuracy. These results of this work will lead to the development of "IMU" state estimation system that supplies current motion information (position and attitude states) that can be used to carry out guidance and control strategies.

\section{References}

[1] Jong-Hyuk Kim, Stuart Wishart and Salah Sukkarieh, "Real-time Navigation, Guidance, and Control of a UAV using Low-cost Sensors", Australian Centre for Field Robotics University of Sydney, NSW 2006, Australia.

[2] Elleithy, M.A., M.O Tantawy, M. E. Ghoniemy, "Development of Six-Degrees of Freedom Model For Surface to Surface Strap down Ballistic Missile" GC-5, Third ASAT Conferences M.T.C. Cairo, Egypt 1989.

[3] George M. Siouris, "Aerospace Avionics Systems" A Modern Synthesis Air Force Institute of Technology 1993.

[4] El-sheikh, G.A., "An Investigation into the performance of strap down Inertial Navigation Systems" Thesis, M.T.C., Cairo, Egypt 1990.

[5] Ibrahim I. Arafa, M. I. Latif, Zhong .H.Yue " Correction of the Flight Path Based on Error Estimation Of SINS Ground Alignment Using Modern Computer Techniques" Beihang University, Beijing 100083,Conferences second Asian workshop of foundations of software. Nanjing China DEC. 2003.

[6] Ibrahim Ismail Arafa, "Analysis and Design of Guidance Navigation and Control Systems for Missile Applications", Beihang University Sep. 2004.

[7] Rodeny E. Stubbs, Whittak H. Huang and Eric Schimtz "Integration of GPS into Ballistic Navigation Solution" Lockheed Martin Astronautics By American Institute of Aeronautics and Astronautics, Inc. 1997.

[8] M. Napier, "Integration of Satellite and Inertial Positioning Systems" Institute of Engineering Surveying and Space Geodesy, University of Nottingham, Vol. 43, 1995.

[9] Ne Eduardo, Durrant-Whyte Hugh. Department of Mechanical and Mechatronic Engineering University of Sydney, 2006, NSW, Australia, 0-8186- 8025-3/97IEEE 1997.

[10] Ye Fuh Jiang Chung Shan Institute of Science and technology, Taiwan "Error Analysis of Analytic Coarse Alignment methods" IEEE Transaction on Aerospace and Electronic, 2. May 1997.

[11] Kayton, M., and Fried, W.R. (1969) "Avionics Navigation Systems"

[12] Huddle, J. R. (1983) "Inertial navigation system error-model considerations in Kalman filtering applications". In C.T. Leondes (Ed.), Control and Dynamics Systems. New York: Academic Press, 20 (1983). 